Case Report

\title{
Intramuscular ganglion of gastrocnemius muscle-unusual presentation of a common tumour
}

\author{
Sanoop Koshy Zachariah*, Gayathri Ajay, Kocheril Sheryl Mathews, John Thomas
}

Department of General, Gastrointestinal \& Laparoscopic Surgery, MOSC Medical College, Kolenchery, India

Received: 24 October 2017

Accepted: 28 November 2017

\section{*Correspondence:}

Dr. Sanoop Koshy Zachariah,

E-mail: skzach@yahoo.com

Copyright: ( ) the author(s), publisher and licensee Medip Academy. This is an open-access article distributed under the terms of the Creative Commons Attribution Non-Commercial License, which permits unrestricted non-commercial use, distribution, and reproduction in any medium, provided the original work is properly cited.

\begin{abstract}
Ganglion cysts (GCs) are one of the most common benign cystic lesions that commonly arise from joints of the wrist. The diagnosis is usually straightforward in these situations. Nevertheless, they have been reported to rarely arise in certain unusual anatomic locations leading to diagnostic difficulties. Here we report an atypical presentation of a ganglion cyst within the gastrocnemius muscle. To the best of our knowledge this is the second case of histologically proven intramuscular ganglion cyst presenting within the gastrocnemius muscle, to be reported in literature.
\end{abstract}

Keywords: Ganglion, Gastrocnemius muscle, Intramuscular ganglion

\section{INTRODUCTION}

Ganglion cysts (GCs) are benign non- neoplastic cystic lesions and are classified as pseudocysts due to lack of a true epithelial lining. The male to female prevalence ratio of ganglion cysts is 1:3. They can occur at any age. The diagnosis is usually straightforward in these situations. Nevertheless, they have been reported to rarely arise in certain unusual anatomic locations leading to diagnostic difficulties. Here we report an atypical presentation of a ganglion cyst within the gastrocnemius muscle.

To the best of our knowledge this is the second case of histologically proven intramuscular ganglion cyst (IMGC) presenting within the gastrocnemius muscle, to be reported in literature.

Nicholson and Freedman had reported the first case of histologically proven IMGC of the gastrocnemius muscle a 53-year-old female. ${ }^{1}$

\section{CASE REPORT}

A 42 year old previously healthy female presented with complaints of a swelling in the right calf of 4 months duration. It was initially not associated with any symptoms. However, for two months, she noticed an increase in the size of the swelling and also developed an aching pain over the calf especially during walking and prolonged standing. She denied any history of trauma in the recent past.

Clinical examination revealed an oval cystic swelling of size $7 \mathrm{~cm} \times 6 \mathrm{~cm}$ on the posteromedial aspect of right leg over proximal expanse of gastrocnemius muscle. The swelling was not tender, pulsatile or compressible with no obvious involvement of the knee joint on physical examination. The knee joint was normal clinically. There was no evidence of associated neural or vascular compression. 
Routine laboratory investigations were within normal limits. An ultrasound scan revealed a cystic lesion in the posteromedial aspect of middle part of right calf with a provisional diagnosis of a baker's cyst.

An MRI scan was performed for better characterization of the lesion which subsequently revealed a well-defined intramuscular $\mathrm{T} 2 \mathrm{~W}$ and $\mathrm{T} 2 \mathrm{~W}$ fat suppression hyperintense cystic lesion with smooth margins and thin septa involving right proximal medial aspect of gastrocnemius muscle with no edema. The size of the cyst was $7.2 \times 4 \times 3 \mathrm{~cm}$. The findings were in favour of an intramuscular ganglion cyst from the right gastrocnemius muscle.

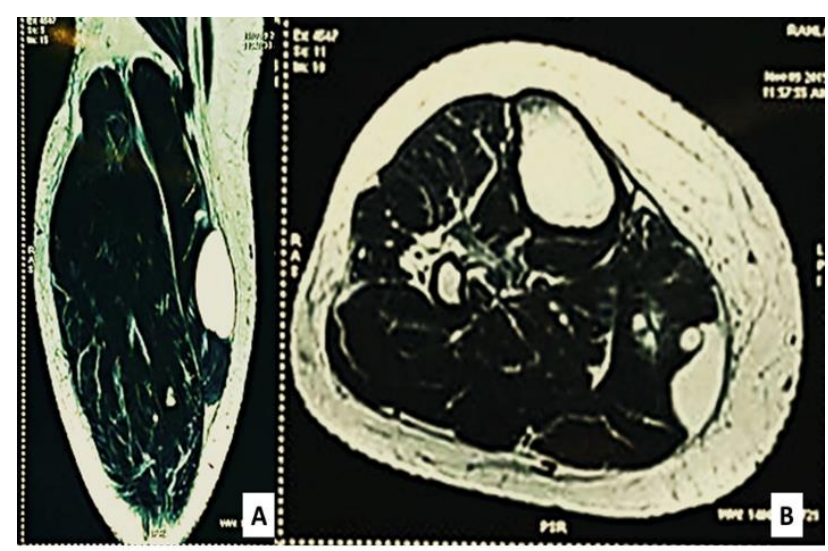

Figure 1: T2-weighted coronal (A) and axial (B) magnetic resonance image showing an intramuscular cyst of the gastrocnemius muscle.

Complete excision of the cyst was done under spinal anaesthesia. The swelling was found closely adherent within the fibres of the gastrocnemius muscle. There was a narrow neck on the proximal aspect of the swelling which was transfixed and excised.

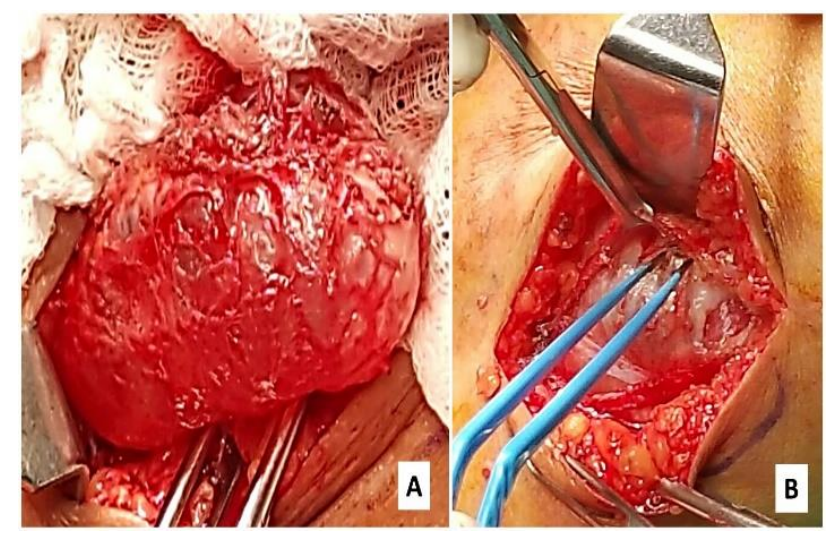

Figure 2: (A and B): Intraoperative image showing the dissection of the intramuscular ganglion lying closely associated and within the fibres of the gastrocnemius muscle.

Histo-pathological evaluation of the excised specimen revealed muscle tissue with a cyst lined by flattened cells and fibrous tissue in the wall which is consistent with ganglion.

\section{DISCUSSION}

The exact etiopathogenesis of GC is not certain. The commonly accepted theory is that they result from secondary myxoid degeneration of the connective tissue that is associated with the joint capsules and the tendon sheaths. ${ }^{2}$ GCS commonly occur on the radial side of the extensor digiti communis tendon on the dorsum, the flexor carpi radialis and the abductor pollicis longus tendons on the volar side of the wrist. ${ }^{3}$

GC rarely arise from cartilage, cruciate ligaments, nerves, muscles and the bone. (intraosseous). In about $30 \%$ of the cases they are isolated cysts implying that they have no communication with adjacent joint capsules. Various theories have been put forward to explain the occurrence of rare Intramuscular ganglion cysts. ${ }^{4-6}$ Some opinion that these are sequestration cysts formed by the seepage of joint fluid from a defect on the surface of a tendon and leading to pooling up within the intramuscular plane forming a fluid like collection or pseudocyst. These kinds of cysts have been termed intramuscular dissecting ganglion cysts. However, others are of the opinion that they can arise from sequestration of extra-articular synovial remnants at the time of joint development or micro-herniation through defects in the joint capsule or tendon sheath which subsequently gets strangulated and cutoff.

IMGC are usually asymptomatic and most often incidentally detected on radiological evaluation for some other condition and in some cases, represents a diagnostic dilemma. The differential diagnosis tendon tear, baker's cyst, bursitis, tenosynovitis. abscess, myxoma, nerve sheath tumour, vascular lesions lipomas and synovial sarcoma.

The preoperative diagnosis is usually based on radiological evaluation. Plain radiographs are often not helpful. Although GCs can be identified on ultrasonography it may be usually insufficient to be able to establish the intricate details of the probable origin of the lesion. MRI is often necessary to study the relations with the joint, adjacent structures and might provide valuable information with regards to its connection with the joint and origin.

Intramuscular ganglion cysts around the knee are a lot more uncommon, with only a few cases reported in the literature, in the gastrocnemius and the semimembranosus, related to the tendon. ${ }^{7}$ The most important differential diagnosis is the baker's cyst which in our case also, was the initial report based on Sonography. Baker's cyst usually arises from the semimembranosus-gastrocnemius bursa. 
Aspiration is not recommended for GC as they have a high chance of recurrence. Surgical excision is the gold standard for management of GC.

\section{CONCLUSION}

Intramuscular ganglion cysts are rare. Magnetic resonance imaging is diagnostic. Ganglion cysts must be considered in the differential diagnosis of cystic lesions in the calf or in relation to the knee joint.

\section{ACKNOWLEDGEMENTS}

Authors would like to appreciate the support of the administration of MOSC medical college Kolenchery, Kerala, India.

Funding: No funding sources

Conflict of interest: None declared

Ethical approval: Not required

\section{REFERENCES}

1. Nicholson L, Freedman H. Intramuscular dissection of a large ganglion cyst into the gastrocnemius muscle. Orthopaedics. 2012;35:e1122-24.

2. Angelides AC, Wallace PF. The dorsal ganglion of the wrist: its pathogenesis, gross and microscopic anatomy, and surgical treatment. J Hand Surg Am. 1976;1:228-35. [PubMed]

3. Nelson CL, Sawmiller S, Phalen GS. Ganglions of the wrist and hand. J Bone Joint Surg Am. 1972;54:1459-64. [PubMed]

4. Garg S, Al-Jabri T, Mutnat S, Moftah F. A giant ganglion cyst of the semimembranosus tendon: a case report. Cases Journal. 2009;2:8305.

5. Yang SW, Teng HP, Tarng YW, Wong CY. Intramuscular ganglion cyst of the quadriceps muscle: Report of a case. Mid Taiwan J Med. 2002;7:193-7.

6. Park S, Jin W, Chun YS, Park SY, Kim HC, Kim $\mathrm{GY}$, et al. Ruptured intramuscular ganglion cyst in the gastrocnemius medialis muscle: sonographic appearance. J Clin Ultrasound. 2009;37:478-81. [PubMed]

7. Kim S, Park J, Choi J. Intratendinous ganglion cyst of the semimembranosus tendon. Brit J Radiology. 2010;83:e79-e82.

Cite this article as: Zachariah SK, Gayathri A, Mathews KS, Thomas J. Intramuscular ganglion of gastrocnemius muscle-unusual presentation of a common tumour. Int Surg J 2018;5:339-41. 